\title{
miR-199a-3p enhances cisplatin sensitivity of ovarian cancer cells by targeting ITGB8
}

\author{
YAJIE CUI $^{1 *}$, FENGQIN WU $^{2 *}$, DEFU TIAN ${ }^{3}$, TING WANG $^{4}$, TIANJIE LU $^{5}$, \\ XIYING HUANG ${ }^{5}$, PEILIAN ZHANG ${ }^{5}$ and LI QIN $^{6}$ \\ ${ }^{1}$ Department of Obstetrics and Gynecology, Xi'an No. 4 Hospital, Xi'an, Shaanxi 710002; \\ ${ }^{2}$ Department of Gynecology, Shangluo Central Hospital, Shangluo, Shaanxi 726000; ${ }^{3}$ Department of General Surgery, \\ Shaanxi Provincial Fourth People's Hospital, Xi'an, Shaanxi 710006; ${ }^{4}$ Reproductive Medicine Center, \\ Northwest Women and Children's Hospital, Xi'an, Shaanxi 710061; Department of Obstetrics and Gynecology, \\ Xi'an No. 1 Hospital, Xi'an, Shaanxi 710002; ${ }^{6}$ Department of Obstetrics and Gynecology, \\ Shaanxi Province People's Hospital, Xi'an, Shaanxi 710033, P.R. China
}

Received August 25, 2017; Accepted January 12, 2018

DOI: $10.3892 /$ or.2018.6259

\begin{abstract}
Drug resistance remains a large obstacle for the treatment of ovarian cancer. miRNAs have been reported to be involved in cisplatin (CDDP) resistance in ovarian cancer. The aim of the present study was to investigate the function and mechanism of miR-199a-3p in the CDDP resistance in ovarian cancer. We found that miR-199a-3p was significantly downregulated in chemoresistant ovarian cancer tissues, as well as CDDP-resistant SKOV3/CDDP cells, compared to chemosensitive carcinomas and SKOV3 cells. Restoration of miR-199a-3p in SKOV3/CDDP cells reduced cell proliferation, G1 phase cell cycle arrest, cell invasion, and increased cell apoptosis, resulting in enhanced CDDP sensitivity, while miR199a-3p inhibition resulted in the opposite effects. Luciferase reporter assay showed that integrin $\beta 8$ (ITGB8), one of the integrins that is involved in the regulation of cell cycle and motility, was a direct target of miR-199a-3p. Overexpression of miR-199a-3p downregulated ITGB8 expression via binding to its 3'-UTR. In addition, overexpression of ITGB8 restored CDDP resistance inhibited by miR-199a-3p. Moreover, orthotopic ovarian cancer mouse model showed that miR-199a-3p enhanced CDDP sensitivity of ovarian cancer in vivo.
\end{abstract}

Correspondence to: Professor Li Qin, Department of Obstetrics and Gynecology, Shaanxi Province People's Hospital, 256 Youyi West Road, Xi'an, Shaanxi 710033, P.R. China

E-mail: qinli_qin_li@163.com

Professor Peilian Zhang, Department of Obstetrics and Gynecology, Xi'an No. 1 Hospital, 30 South Street Powder Lane, Xi'an, Shaanxi 710002, P.R. China

E-mail: peilian_zhang@21cn.com

*Contributed equally

Key words: miR-199a-3p, CDDP sensitivity, ovarian cancer, ITGB8
Therefore, our results indicate that miR-199a-3p enhances CDDP sensitivity of ovarian cancer cells through downregulating ITGB8 expression, and miR-199a-3p may serve as a therapeutic target for the treatment of ovarian cancer patients with CDDP-resistance.

\section{Introduction}

Ovarian cancer is the leading cause of death among women (1). Currently, the main treatment for ovarian cancer is surgical excision followed by combination chemotherapy. Cisplatin (CDDP) is the most effective chemotherapy drug for the treatment of ovarian cancer (2). However, CDDP chemoresistance remains a large obstacle for the successful treatment of ovarian cancer (3). Therefore, it is important to explore the molecular basis of CDDP resistance to improve the success rate of chemotherapy.

MicroRNAs (miRNAs) are noncoding, single stranded RNAs that regulate gene expression by binding the 3'-untranslated regions (3'-UTRs) of target mRNAs (4). Ovarian cancer patients who were resistant to chemotherapy frequently had different miRNA expression patterns compared to those of the sensitive patients (5). For example, microarray identified that let-7i was the first miRNA associated with CDDP resistance of ovarian cancer (6). Moreover, miR-595 (7), miR-186 (8) and miR-141 (9) increased the sensitivity of ovarian cancer cells to CDDP by targeting multiple signaling pathways, whereas miR-130a and miR-374a contribute to CDDP resistance in ovarian cancer (10). However, the role and mechanism of miRNA involved in CDDP resistance in ovarian cancer remains largely unknown.

In the present study, we explored the expression pattern and biological effects of miR-199a-3p in CDDP chemosensitivity in ovarian cancer. We found that miR-199a-3p was significantly downregulated in CDDP-resistant ovarian cancer tissues and cell lines. Overexpression of miR-199a-3p results in enhanced CDDP sensitivity. ITGB8 was a direct target of miR-199a-3p and ectopic expression of ITGB8 alleviated the 
CDDP sensitivity. Importantly, miR-199a-3p also enhanced CDDP sensitivity in ovarian cancer in vivo. Collectively, our findings suggest that miR-199a-3p plays a critical role in regulating CDDP chemosensitivity by targeting ITGB8 in ovarian cancer.

\section{Materials and methods}

Tissue specimens, cell lines and cell culture. Thirty cases of CDDP-sensitive and 28 cases of CDDP-resistant human ovarian cancer tissues were collected from the Department of Obstetrics and Gynecology, Xijing Hospital, The Fourth Military Medical University between Jan 2008 and Dec 2012. Tissues were frozen in liquid nitrogen and stored at $-80^{\circ} \mathrm{C}$ until use. All patients were treated with the standard care of platinum-based therapy after surgery at least for 6 months. Platinum resistance or platinum sensitivity was defined as a relapse or progression within 6 months or 6 months after the last platinum-based chemotherapy. The present study was approved by the Ethics Committee of Xi'an No. 1 Hospital and written informed consent was obtained from each patient. Clinicopathological characteristics of chemoresistant and chemosensitive ovarian cancer patients are shown in Table I. Human ovarian cancer cell line SKOV3 was purchased from Shanghai Institute of Cell Biology (Shanghai, China). Cells were maintained in RPMI-1640 supplemented with 10\% FBS at $37^{\circ} \mathrm{C}$ in a humidified incubator with $5 \% \mathrm{CO}_{2}$.

Ethics approval. All procedures performed in studies involving human participants were in accordance with the ethical standards of the institutional and/or national research committee and with the 1964 Helsinki declaration and its later amendments or comparable ethical standards. All applicable international, national, and/or institutional guidelines for the care and use of animals were followed. All applicable international, national, and/or institutional guidelines for the care and use of animals were followed.

Establishment of CDDP-resistant ovarian cancer cell lines. The CDDP-resistant ovarian cancer cell lines were induced using progressive concentration of CDDP as previously described (11). Briefly, SKOV3 cells were first treated with $0.5 \mu \mathrm{g} / \mathrm{ml}$ of CDDP, CDDP was withdrawn $48 \mathrm{~h}$ later and cells were cultured without CDDP until they recovered. The same treatment was performed with CDDP, the concentration was gradually increased to $1,2,3,4$ and finally to $6 \mu \mathrm{g} / \mathrm{ml}$. When the induced cells still survived in $6 \mu \mathrm{g} / \mathrm{ml}$ of CDDP, the cells were confirmed to be CDDP-resistant and named SKOV3/CDDP.

Cell transfection. miR-199a-3p mimics, miR-199a-3p inhibitor and negative control (NC) were designed and synthesized by GeneBioPharma (Shanghai, China). Transient transfection was performed using HiPerFect Transfection Reagent (Qiagen, Hilden, German) as instructed by the manufacturer. Full-length ITGB8 cDNA was amplified and cloned into the pcDNA3.1 vector, primer for amplication ITGB8 was as follows: forward, 5'-CCGGAATTCATGTCCCGCCCACGC TG-3' and reverse, 5'-CGGGATCCTTAATTAATCCAGTCA TCT-3'. Transfection was performed using Lipofectamine LTX (Thermo Fisher Scientific, Waltham, MA, USA) according to the manufacturer's protocol. Transfection efficiencies were assessed using qRT-PCR.

Cytotoxicityassay. The CDDP cytotoxicity was measured using a Cell Counting Kit-8 (CCK-8) assay (Dojindo Laboratories, Kumamoto, Japan). Briefly, cells were collected and reseeded in triplicate in $200 \mu \mathrm{l}$ of medium in a 96 -well plate at $5 \times 10^{3}$ cells/ well with $0,0.0625,0.125,0.25,0.05,1,2,4,8 \mu \mathrm{g} / \mathrm{ml}$ of CDDP. Seventy-two hours after culture, $20 \mu 1$ of CCK- 8 was added and cultured for additional $4 \mathrm{~h}$. The absorbance at $490 \mathrm{~nm}$ was measured using microplate reader (Bio-Tek, Winooski, VT, USA). The concentration of CDDP that caused 50\% inhibition of ovarian cancer cell activity was defined as $\mathrm{IC}_{50}$. The inhibition rate was calculated using the following formula: Inhibition rate $(\%)=\left(1-\mathrm{OD}_{\text {treated }} / \mathrm{OD}_{\text {control }}\right) \times 100 \%$.

Cell apoptosis assay. Cell apoptosis was evaluated by flow cytometry using Annexin V-FITC/PI detection kit (KeyGen Biotech Co., Nanjing, China) according to the manufacturer's instructions. Data were acquired on a FACSCalibur flow cytometer (BD Biosciences, San Jose, CA, USA) and analyzed using FlowJo software (Tree Star, Inc., Ashland, OR, USA).

Cell cycle analysis. Cell cycle was measured using propidium iodide (PI) staining. Briefly, $24 \mathrm{~h}$ post transfection, cells were collected and reseeded in triplicate in a 6-well plate at $1 \times 10^{5}$ cells/well, cells were harvested $24 \mathrm{~h}$ later, and washed with PBS, fixed in ice-cold $70 \%$ ethanol overnight. Cells were then washed with ice-cold PBS, resuspended in $500 \mu \mathrm{l}$ of a PI master mix containing $50 \mu \mathrm{g} / \mathrm{ml}$ PI and $100 \mu \mathrm{g} / \mathrm{ml}$ RNAse A (both from Sigma, St. Louis, MO, USA) diluted in PBS and incubated for $30 \mathrm{~min}$ in the dark at $37^{\circ} \mathrm{C}$. Cell cycle was measured using BD FACSCalibur flow cytometer (BD Biosciences, San Diego, CA, USA), and analyzed using FlowJo software (Tree Star, Inc.).

Wound healing assay. SKOV3 or SKOV3/CDDP cells were seeded into 6-well plates at $3 \times 10^{5}$ cells/well and cultured until confluent. A P200 pipette tip was used to make a straight scratch, and then incubated for $48 \mathrm{~h}$. The gap width of scratch re-population was recorded under the microscope.

Cell invasion assay. Cell migration assays were performed using 24-well Transwells (8- $\mu \mathrm{m}$ pore size; Millipore Corporation, Billerica, MA, USA) pre-coated with $100 \mu 1$ of Matrigel (BD Pharmingen, San Jose, CA, USA). Briefly, miRNA transfected or control cells were suspended in $200 \mu \mathrm{l}$ RPMI-1640 medium then added to the upper chamber. A volume of $600 \mu \mathrm{l}$ of RPMI-1640 containing 10\% FBS was placed in the lower chamber. After incubating for $48 \mathrm{~h}$, the Matrigel and the cells remaining in the upper chamber were removed using cotton swabs. Cells on the lower surface of the membrane were fixed with $4 \%$ paraformaldehyde and stained with Giemsa (Sigma-Aldrich, St. Louis, MO, USA). Five microscopic fields (at a magnification of x200) were used to count and photograph the cells.

RNA extraction and real-time quantitative PCR. Total RNA were extracted from ovarian cancer tissues, transfected SKOV3 cells or xenografts using the RNeasy Mini kit (Qiagen, 
Table I. Clinicopathological characteristics of chemoresistant and chemosensitive ovarian cancer patients.

\begin{tabular}{|c|c|c|c|c|c|}
\hline Characteristics & Cases & Chemosensitive, n (\%) & Chemoresistant, n (\%) & $\chi^{2}$ & P-value \\
\hline Age (years) & & 34 & 24 & & \\
\hline$<50$ & 39 & $23(59.0)$ & $16(41.0)$ & 0.793 & 0.459 \\
\hline$\geq 50$ & 19 & $11(57.8)$ & $8(42.2)$ & & \\
\hline \multicolumn{6}{|l|}{ FIGO stage } \\
\hline I & 14 & $10(71.40)$ & $4(28.6)$ & 1.926 & 0.503 \\
\hline II & 18 & $10(55.6)$ & $8(44.4)$ & & \\
\hline III & 17 & $9(52.9)$ & $8(47.1)$ & & \\
\hline IV & 9 & $5(55.6)$ & $4(44.4)$ & & \\
\hline \multicolumn{6}{|c|}{ Histological grade } \\
\hline G1 & 6 & $4(66.7)$ & $2(33.7)$ & 0.732 & 0.625 \\
\hline $\mathrm{G} 2$ & 20 & $11(55.0)$ & $9(45.0)$ & & \\
\hline G3 & 32 & $19(59.3)$ & $13(40.7)$ & & \\
\hline \multicolumn{6}{|l|}{ Histology } \\
\hline Serous & 30 & $19(63.3)$ & $11(36.7)$ & 2.037 & 0.561 \\
\hline Mucinous & 11 & $6(54.5)$ & $5(35.5)$ & & \\
\hline Endometrioid & 10 & $6(60)$ & $4(40)$ & & \\
\hline Clear cell & 3 & $1(33.3)$ & $2(66.7)$ & & \\
\hline Anaplastic & 4 & $2(50.0)$ & $2(50.0)$ & & \\
\hline \multicolumn{6}{|c|}{ Lymph node metastasis } \\
\hline Yes & 26 & $11(42.3)$ & $15(57.7)$ & 2.453 & 0.471 \\
\hline No & 32 & $23(71.8)$ & $9(28.2)$ & & \\
\hline
\end{tabular}

FIGO, International Federation of Gynecology and Obstetrics.

Shanghai, China) following the manufacturer's instructions. The concentration and quality of the RNA was examined by NanoDrop 2000 (Thermo Fisher Scientific). Total RNA of $1 \mu \mathrm{g}$ was reversely transcribed into cDNA using miScript II RT kit (Qiagen). cDNA was then used for real-time PCR with miScript SYBR-Green PCR kit (Qiagen). The amplification was performed on an Applied Biosystems 7500 Fast Real-Time PCR System (Applied Biosystems, Foster City, CA, USA), with a first step at $95^{\circ} \mathrm{C}$ for $3 \mathrm{~min}$ and then 40 cycles with $95^{\circ} \mathrm{C}$ for $15 \mathrm{sec}, 60^{\circ} \mathrm{C}$ for $5 \mathrm{sec}, 68^{\circ} \mathrm{C}$ for $30 \mathrm{sec}$ with a fluorescent reading at the end of this step. Primers used were as follows: RT primes for miR-199a-3p, 5'-CTCAACTGG TGTCGTGGAGTCGGCAATTCAGTTGAGTAACCAAT-3'; miR-199a-3p forward, 5'-ACACTCCAGCTGGGACAGTAG TCTGCACAT-3' and reverse, 5'-TGGTGTCGTGGAGTC G-3'; U6 forward, 5'-ATTGGAACGATACAGAGAAGATT-3' and reverse, 5'-GGAACGCTTCACGAATTTG-3'; ITGB8 forward, 5'-CCCGTGACTTTCGTCTTGGA-3' and reverse, 5'-CCTTTCGGGGTGGATGCTAA-3'; $\beta$-actin forward, 5'-CGTCTTCCCCTCCATCGT-3' and reverse, 5'-GCCTCG TCGCCCACATAG-3'. The relative expression of the gene was quantified using the comparative $2^{-\Delta \Delta \mathrm{Ct}}$ method relative to the appropriate housekeeping gene.

Protein extraction and western blotting. Protein was extracted from transfected cells or xenografts using radioimmunoprecipitation assay (RIPA) lysis buffer (Beyotime Biotechnology,
Shanghai, China). Protein concentration was assessed using a BCA protein assay kit (KeyGen Biotech Co.). Protein $(50 \mu \mathrm{g})$ was separated by $10 \%$ SDS-PAGE, then transferred onto polyvinylidene fluoride membranes (Millipore Corporation, Bedford, MA, USA). The membrane was washed in $0.1 \%$ Tween-20 in Tris-buffered saline solution (TBST), and blocked using 5\% non-fat milk. The membrane was incubated with primary antibodies against ITGB8 (\#88300) and $\beta$-actin (\#8457) (both from Cell Signaling Technology, Inc., Danvers, MA, USA) overnight at $4^{\circ} \mathrm{C}$. After washing 3 times, the membrane was incubated with horseradish peroxidaseconjugated secondary antibody for $1 \mathrm{~h}$ at room temperature in a dilution of 1:2,000. Protein bands were visualized with ECL $^{\text {тм }}$ Western Blotting Detection Reagents (Amersham; GE Healthcare, Chalfont, UK) and captured using Bio-Rad Imaging Systems (Bio-Rad).

Dual-luciferase reporter assay. Two 3'-UTR of ITGB8 containing miR-199a-3p binding sequences was amplified by PCR from genomic DNA. The PCR product was cloned into psiCHECK-reporter vector (Promega, Madison, WI, USA). Mutant construct of the ITGB8 3'-UTR-2, containing a mutated sequence within the miR-199a-3p target site was generated using a site-directed mutagenesis kit (Takara, Tokyo, Japan). psiCHECK-ITGB8-control or psiCHECK-ITGB8-3'-UTR-1 or 2, or psiCHECK-ITGB8-3'-UTR-2 Mut (500 ng) and $50 \mathrm{nM}$ miR-199a-3p mimics or negative control were co-transfected 
A

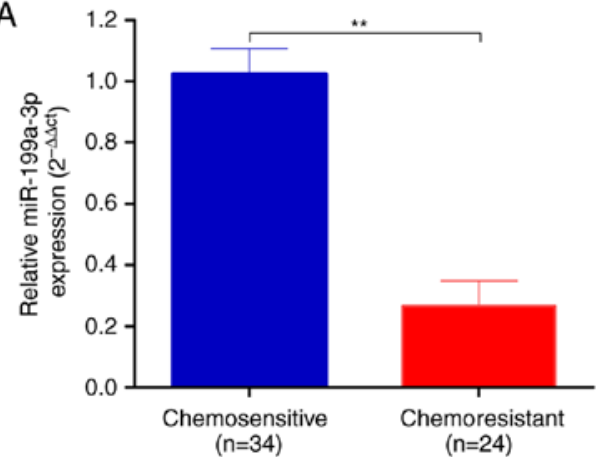

B

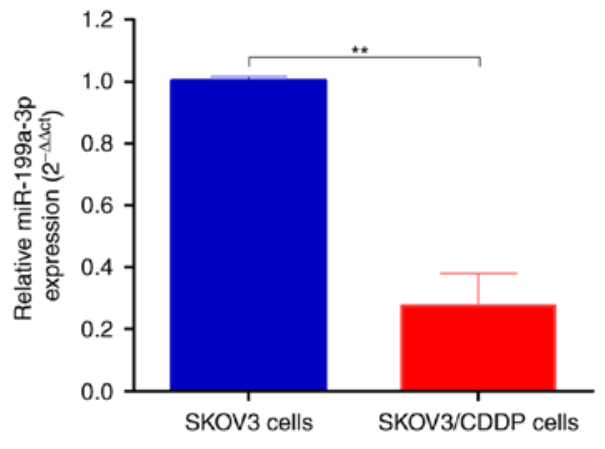

Figure 1. miR-199a-3p is downregulated in cisplatin-resistant ovarian cancer tissues and cell lines. (A) Total RNA was extracted from chemosensitive and chemoresistant ovarian cancer tissues, the miR-199a-3p expression was determined by real-time PCR. (B) miR-199a-3p expression in CDDP-resistant SKOV3/CDDP and normal SKOV3 cells was determined by real-time PCR. Data shown are mean $\pm \mathrm{SD},{ }^{* *} \mathrm{P}<0.01$ (Student's t-test).

into SKOV3/CDDP cells using Lipofectamine LTX. Luciferase signals were measured $48 \mathrm{~h}$ after transfection using a Dual-Luciferase Reporter Assay kit (Promega).

Orthotopic mouse ovarian cancer model. Orthotopic mouse ovarian cancer model was generated in nude mice (female, 6-8 weeks old) as previously described (12). Briefly, mice were anesthetized with pentobarbital, a $1.5 \mathrm{~cm}$ dorsal incision was performed in the kidney region, the left ovarian bursa was exposed and injected with $1 \times 10^{6} \mathrm{SKOV} 3 / \mathrm{CDDP}$ or SKOV3/CDDP/miR-199a-3p cells in $50 \mu 1$ PBS (n=5/group). Animals were euthanized at 10 weeks, tumors were harvested and photographed. The number of peritoneal metastasis was recorded. The mouse experiments were approved by the Institutional Animal Care and Use Committee of Xi'an No. 1 Hospital.

Statistical analysis. Data are shown as means \pm SD. Differences between groups were evaluated using the Student's t-test or one-way ANOVA. GraphPad Prism version 6.00 (GraphPad Software, San Diego, CA, USA) was used for all analyses. $\mathrm{P}<0.05$ was considered as statistical significance.

\section{Results}

miR-199a-3p is downregulated in cisplatin-resistant ovarian cancer tissues and cell lines. Total RNA was extracted from chemosensitive $(n=34)$ and chemoresistant $(n=24)$ ovarian cancer tissues. As shown in Fig. 1A, miR-199a-3p was significantly decreased in chemoresistant ovarian cancer tissues compared to chemosensitive carcinomas $\left(2^{-\Delta \Delta C t}, 0.271 \pm 0.037\right.$ vs. $1.006 \pm 0.124, \mathrm{P}<0.01)$.

We then established the CDDP-resistant SKOV3/CDDP cells by progressive concentration of CDDP induction as described in Materials and methods. The cell proliferation, metastasis and invasion capability of CDDP-resistant ovarian cancer SKOV3/CDDP cell lines were tested. The $\mathrm{IC}_{50}$ value of CDDP for SKOV3 cells was 0.75 and $2.03 \mu \mathrm{g} / \mathrm{ml}$ for SKOV3/CDDP cells. In agreement with the ovarian cancer tissues, the expression level of miR-199a-3p was significantly downregulated in CDDP resistant SKOV3/CDDP cells compared to SKOV3 cells $\left(2^{-\Delta \Delta C t}, 0.07 \pm 0.01\right.$ vs. $1.001 \pm 0.042$, $\mathrm{P}<0.01$; Fig. 1B).
miR-199a-3p enhances the sensitivity of ovarian cancer cell lines to cisplatin. To determine whether miR-199-3p contributed to cisplatin sensitivity of ovarian cancer cells, we transfected the SKOV3/CDDP cells with miR-199-3p mimic, and SKOV3 cells with miR-199-3p inhibitor, respectively. We found that inhibition of miR-199-3p reduced the inhibition rate of CDDP $(0.75 \mu \mathrm{g} / \mathrm{ml})$ in SKOV3 cells, compared to the negative controls, whereas miR-199a-3p mimics improved the toxicity of CDDP $(2.03 \mu \mathrm{g} / \mathrm{ml})$ in SKOV3/CDDP cells (Fig. 2A and B). Apoptosis of miR-199a-3p-overexpressing SKOV3/CDDP cells was significantly increased compared with the negative control, while miR-199a-3p inhibition could decrease the apoptosis rate in SKOV3 cells (Fig. 2C and D). Cell cycle assay indicated that ectopic miR-199a-3p resulted in significantly increased G1 phase but a reduced S phase in SKOV3/CDDP cells compared to the negative controls, while miR-199a-3p inhibition showed the opposite effect (Fig. 2E and F). In addition, Transwell assay showed that miR-199a-3p overexpression markedly suppressed the capacity for cell migration in SKOV3/CDDP cells, while miR-199a-3p inhibition promoted cell migration in SKOV3 cells (Fig. 2G and H). These results indicate that miR-199a-3p enhanced the sensitivity of ovarian cancer cell lines to CDDP.

ITGB8 is a direct target of miR-199a-3p. The computer-aided algorithms (TargetScan Release 5.2, http://www.targetscan.org; and PicTar, (http://pictar.mdc-berlin.de/cgi-bin/new_PicTar_ vertebrate.cgi) predicted that integrin $\beta 8$ (ITGB8), one of the integrins involved in the regulation of cell cycle and motility, was a potential target gene of miR-199a-3p (Fig. 3A). There were two putative binding sites for miR-199a-3p on ITGB8 3'-UTR, one located at position 203-209 (3'-UTR-1), and the other located at position 729-735 of ITGB8 3 '-UTR (3'-UTR-2) (Fig. 3A). Importantly, the ITGB8 level was also upregulated in SKOV3/CDDP cells compared to SKOV3 cells (Fig. 3B). Luciferase assay showed that the luciferase activities decreased significantly in the ITGB8 3'-UTR-2-transfected cells when co-transfected with miR-199a-3p, while ITGB8 3'-UTR-1 transfected cells had no effect on the luciferase activities (Fig. 3C). In addition, the inhibition of luciferase activities by miR-199a-3p was abolished when the ITGB8 3'-UTR-2 site was mutated (Fig. 3D). miR-199a-3p overexpression reduced the ITGB8 mRNA and protein levels in SKOV3/CDDP 

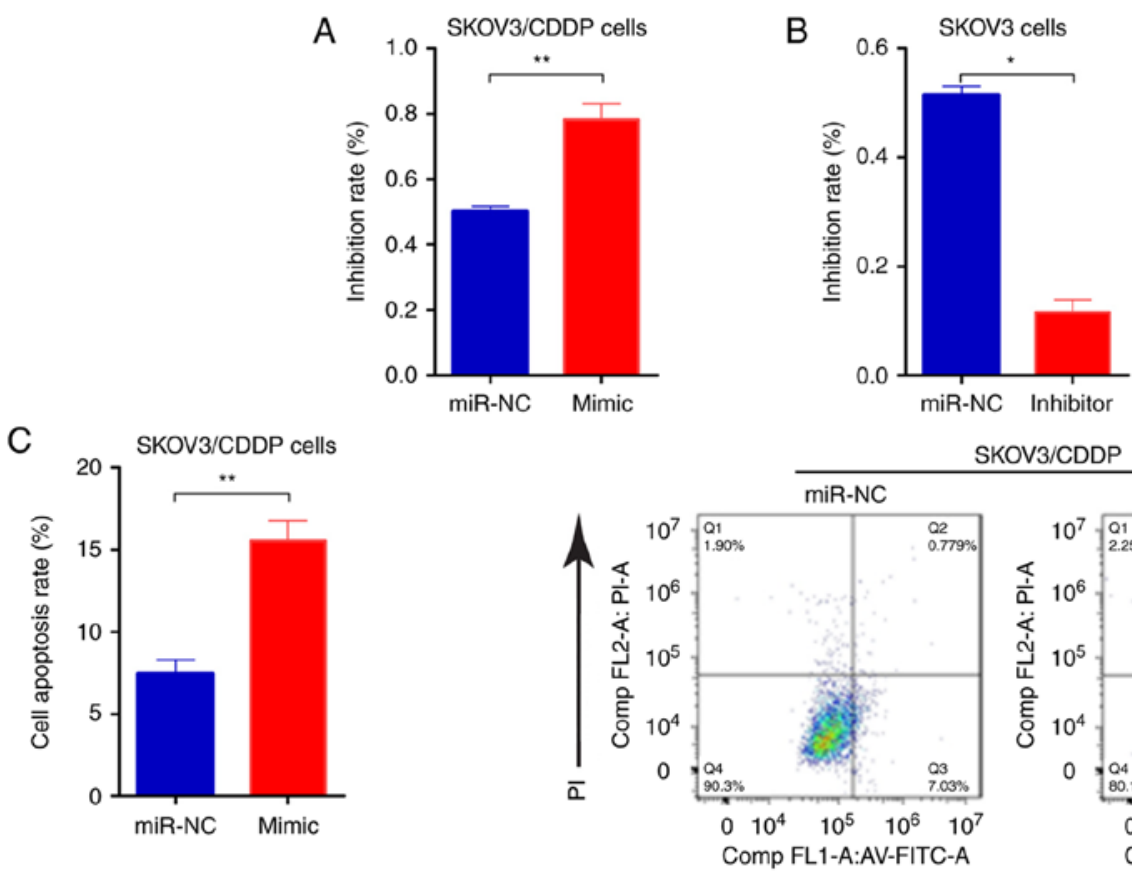

SKOV3/CDDP
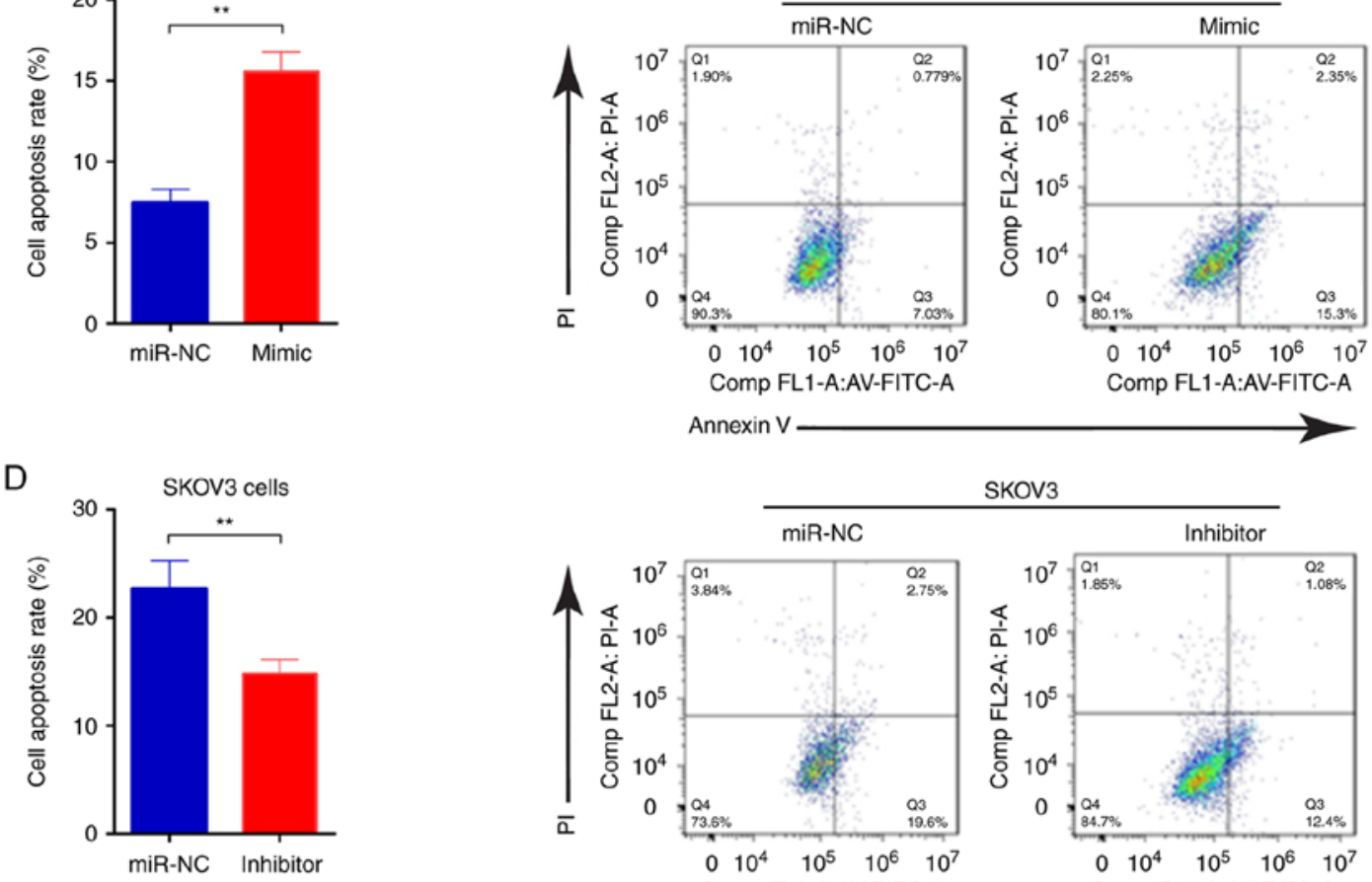

Annexin V

SKOV3

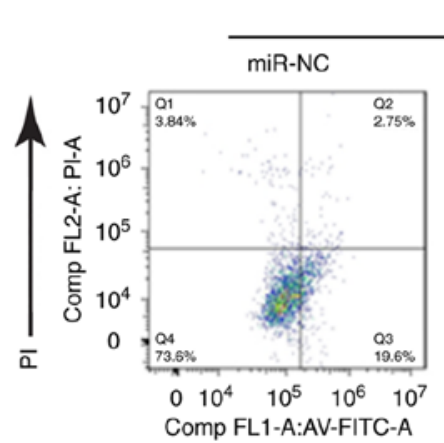

Inhibitor

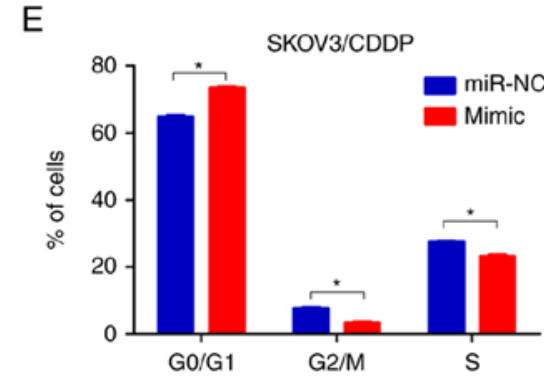

Annexin V
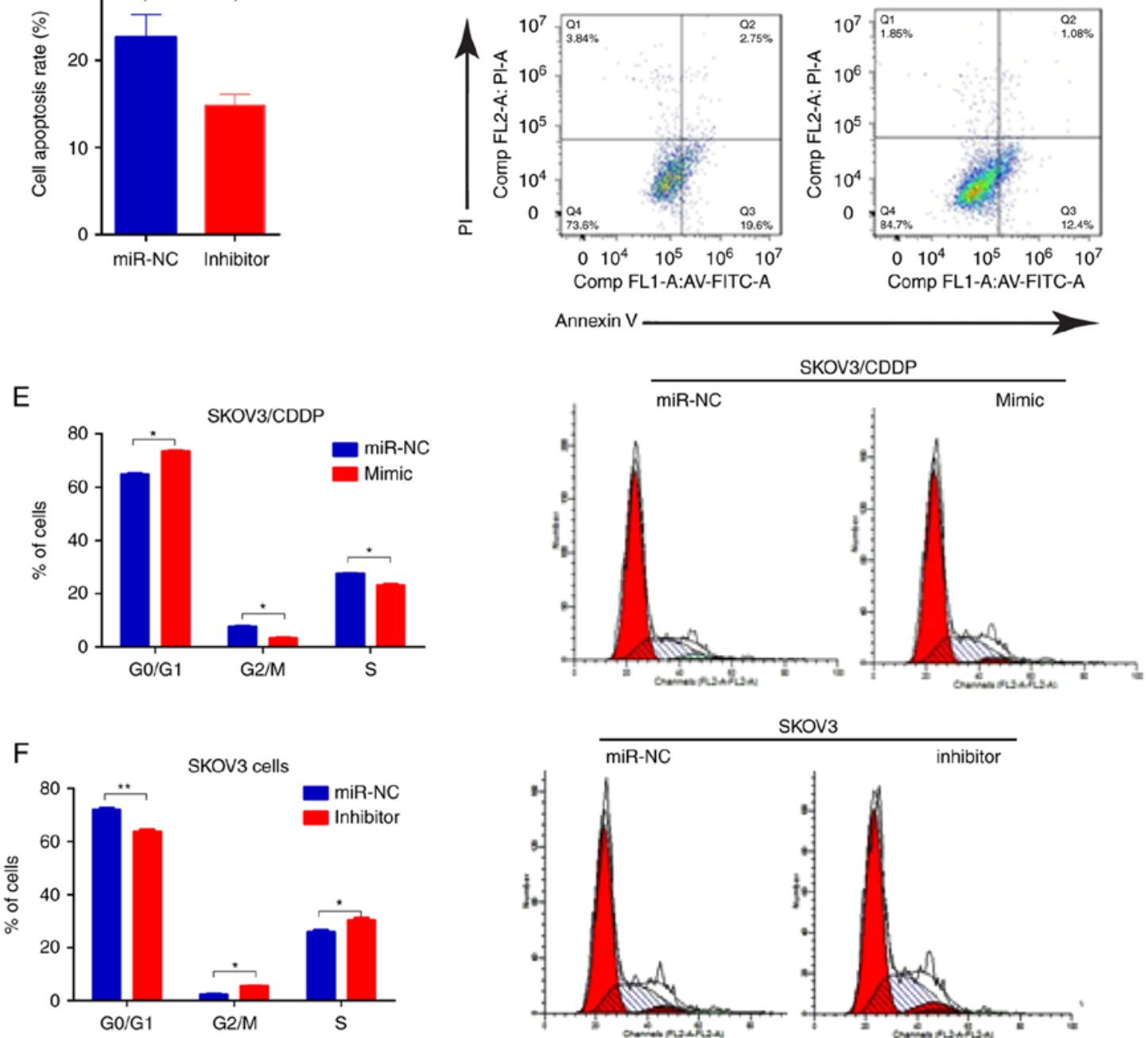

Figure 2. miR-199a-3p enhances the sensitivity of ovarian cancer cell lines to cisplatin. (A and B) Effect of miR-199a-3p mimic or inhibitor on cell proliferation in SKOV3/CDDP cells was detected by CCK-8 assay, the concentration of CDDP used for SKOV3 or SKOV3/CDDP treatment was 0.75 or $2.03 \mu \mathrm{g} / \mathrm{ml}$, respectively. (C and D) Cell apoptosis was detected by Annexin V-FITC/PI detection kit. (E and F) Cell cycle distribution was detected by PI staining and flow cytometry. 

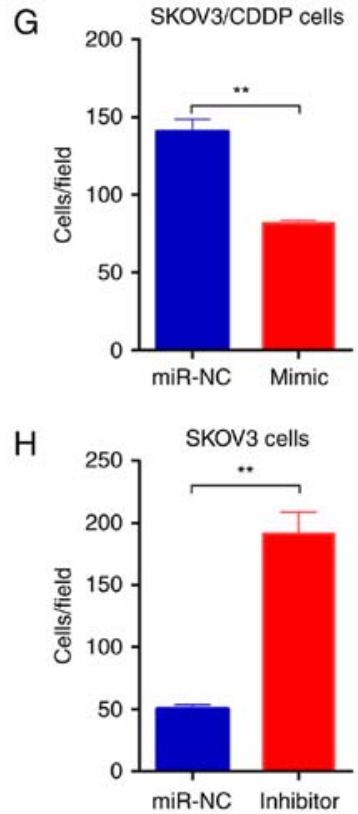
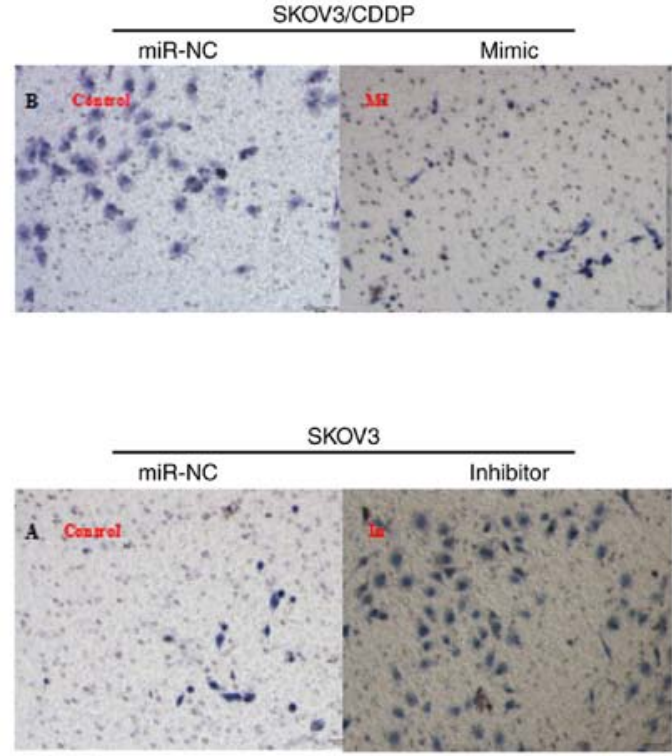

Figure 2. Continued. (G and H) Cell invasion was detected by Transwell assay. Data shown are mean $\pm \mathrm{SD},{ }^{*} \mathrm{P}<0.05,{ }^{* *} \mathrm{P}<0.01$, compared with miR-NC (Student's t-test).

\section{A Position 203-209 of ITGB8 3'-UTR 5' AGAAAAAUGUGUCUUACUACUGU hsa-miR-199a-3p Position 729-735 of ITGB8 3'-UTR hsa-miR-199a-3p \\ 3'AUUGGUUACACGUCUGAUGACA \\ 5'AUUAAGUGC UAAGUACUACUGC 3'AUUGGUUACACGUCUGAUGACA}

B

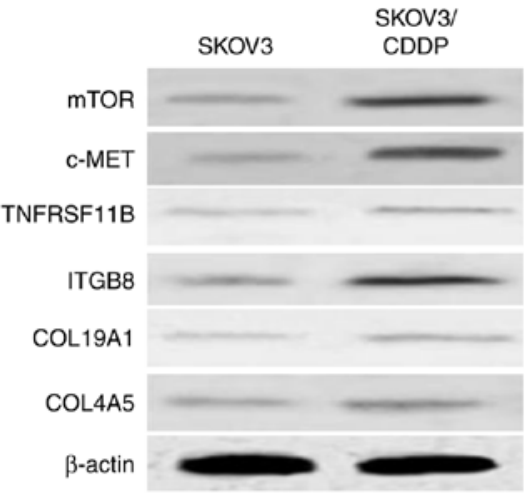

D

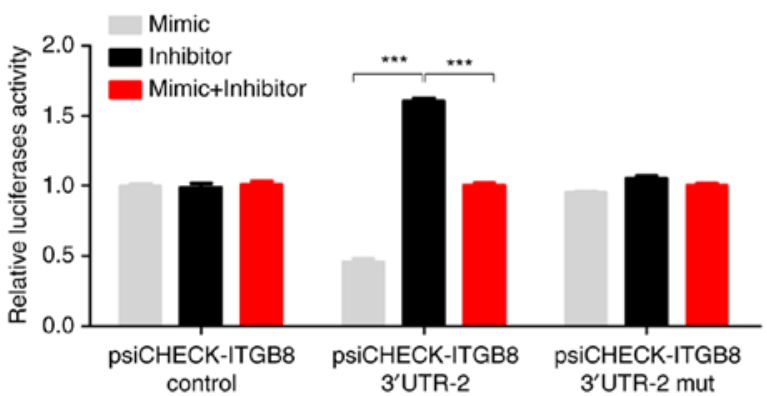

C psiCHECK-control+mimic

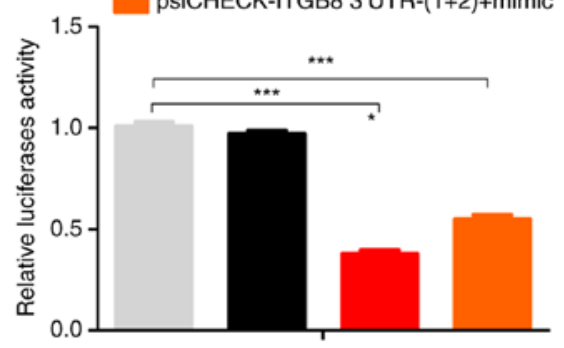

E

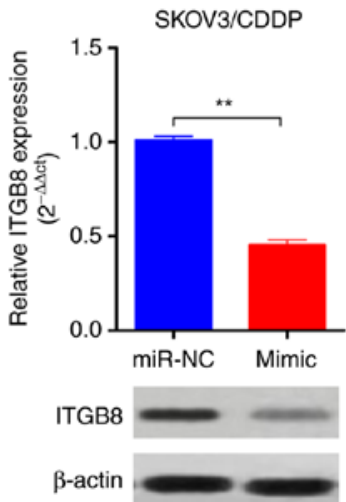

Figure 3. ITGB8 is a direct target of miR-199a-3p. (A) Sequence alignment of two putative miR-199a-3p binding sites in the 3'-UTR of ITGB8. (B) The protein levels of ITGB8 in SKOV3/CDDP and SKOV3 cells examined by western blotting. (C) Relative luciferase activities of plasmids carrying ITGB8 3'-UTR-1 or ITGB8 3'-UTR-2, or both in SKOV3/CDDP cells co-transfected with miR-199a-3p mimics. Data shown are mean \pm SD, ${ }^{* * *}$ P $<0.001$, compared with control mimic (one-way ANOVA with Dunett's test). (D) Relative luciferase activities of plasmids carrying WT or mutant of ITGB8 3'-UTR-2 in SKOV3/CDDP cells co-transfected with miR-199a-3p mimics or miR-199a-3p inhibitor. Data shown are mean $\pm \mathrm{SD},{ }^{* * *} \mathrm{P}<0.01$, compared with inhibitor (one-way ANOVA with Dunett's test). (E) The expression levels of ITGB8 in SKOV3/CDDP cells were examined by real-time PCR and western blotting. Data shown are mean \pm SD, $\mathrm{P}<0.01$, compared with miR-NC (Student's t-test). 


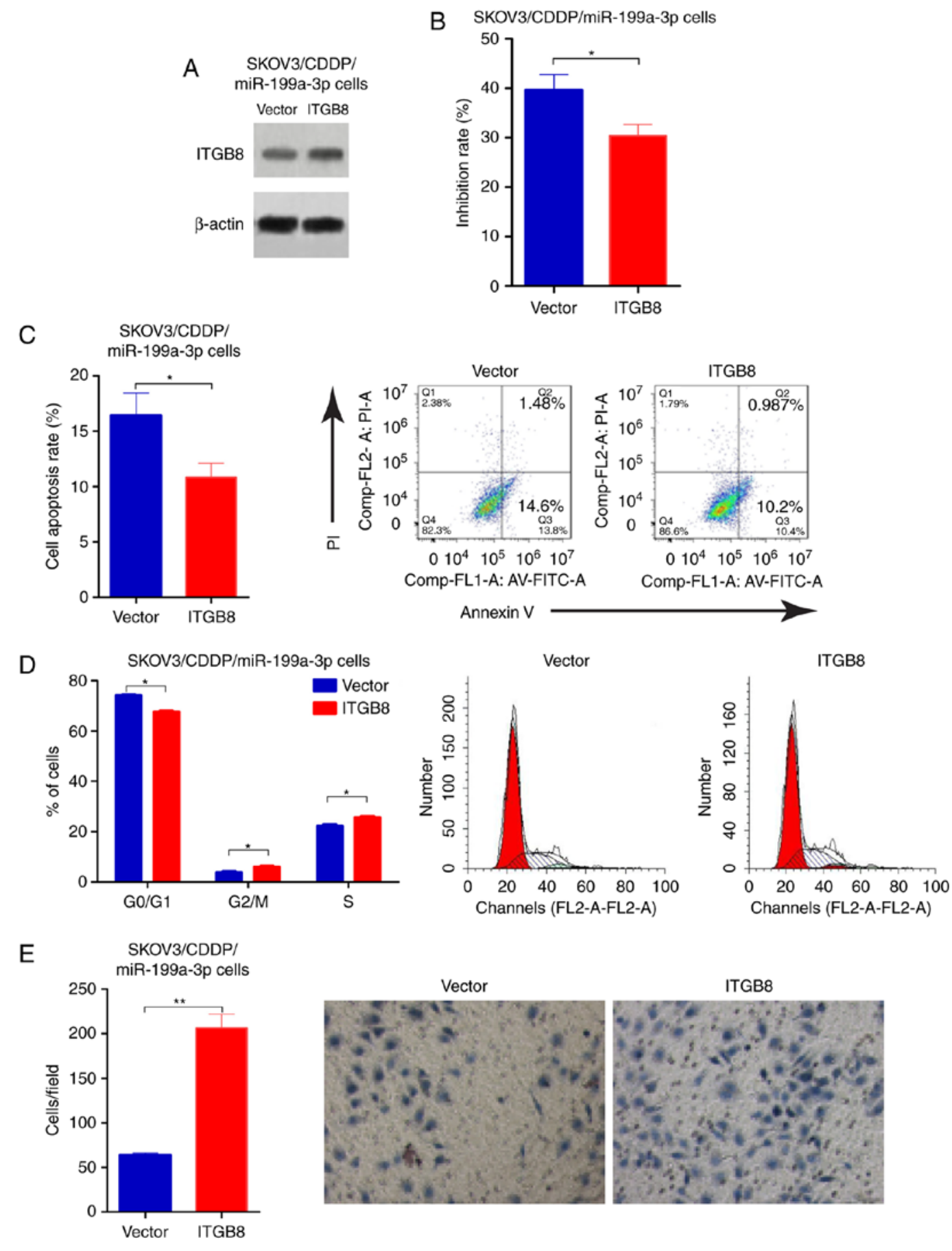

Figure 4. Overexpression of ITGB8 enhances CDDP resistance in miR-199a-3p overexpressed ovarian cancer cells. SKOV3/CDDP/miR-199a-3p cells were transfected with pcDNA3.1- ITGB8 plasmid. (A) The transfection efficiency was validated by western blotting. (B) Cell proliferation was detected by CCK-8 assay. (C) Cell apoptosis was detected by Annexin V-FITC/PI detection kit. (D) Cell cycle distribution was detected by PI staining and flow cytometry. (E) Cell invasion was measured using the Transwell assay. Data shown are the representative images and quantitative data. Data shown are mean $\pm \mathrm{SD}$, ${ }^{*} \mathrm{P}<0.05$, ${ }^{* *} \mathrm{P}<0.01$, compared with vector (Student's t-test).

cells (Fig. 3E). These results indicate that ITGB8 is a functional direct target of miR-199a-3p in ovarian cancer cells.

Overexpression of ITGB8 enhances CDDP resistance in miR-199a-3p overexpressed ovarian cancer cells. To determine the functional involvement of ITGB8 in miR-199a-3p-mediated CDDP resistance, miR-199a-3p-overexpression SKOV3/CDDP cells were transfected with empty vector or ITGB8 plasmid, the transfection efficiency was validated by western blotting (Fig. 4A). ITGB8 overexpression enhances the CDDP resistance that was inhibited by miR-199a-3p (Fig. 4B). Cell apoptosis was reduced in SKOV3/CDDP/miR-199a-3p cells with ITGB8 overexpression compared to the cell line transfected with empty vector (Fig. 4C). In addition, ectopic ITGB8 resulted in significantly decreased G1 phase, but an increased $\mathrm{S}$ phase in SKOV3/CDDP/miR-199a-3p cells compared to the vector controls (Fig. 4D). Transwell assay showed that ITGB8 overexpression markedly suppressed the capacity for cell migration in SKOV3/CDDP/miR-199a-3p cells compared to the vector controls (Fig. 4E). These results demonstrated that 

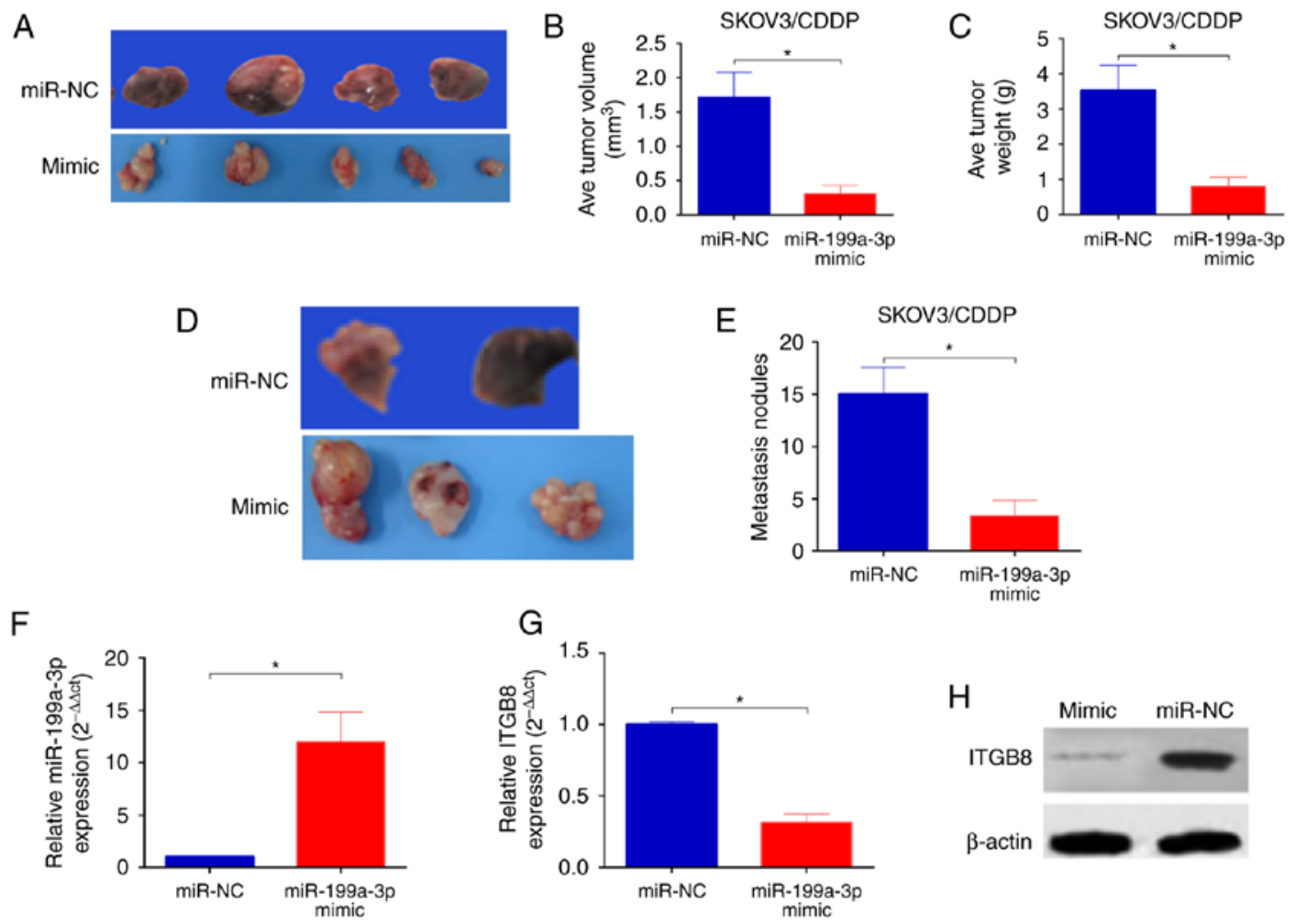

Figure 5. miR-199a-3p enhances CDDP sensitivity of ovarian cancer in vivo. (A) Representative images of tumor xenografts, (B) the tumor volumes and (C) the tumor weight were measured at 10 weeks. (D) Representative images of peritoneal metastasis nodules, and the number of peritoneal metastasis (E) in the two groups. (F-H) The expression of miR-199a-3p and IGTB8 in the xenografts were examined by q-PCR and western blotting. Data shown are mean \pm SD, ${ }^{*} \mathrm{P}<0.05$, compared with miR-NC (Student's t-test).

miR-199a-3p regulated cisplatin sensitivity in ovarian cancer cells by via suppression of ITGB8.

miR-199a-3p enhances CDDP sensitivity of ovarian cancer in vivo. To determine whether the delivery of miR-199a-3p in vivo enhanced the cytotoxicity of CDDP we established an orthotopic ovarian cancer model in nude mice by injecting miR-199a-3p- or miR-NC-overexpression SKOV3/CDDP cells into the ovary of mice. Ten weeks after injection, significantly reduced tumor sizes and lower tumor weight were observed in mice injected with SKOV3/CDDP/miR-199a-3p cells in comparison with those of mice injected with SKOV3/CDDP/miR-NC (Fig. 5A-C). In addition, the number of peritoneal metastasis was also markedly decreased in mice injected with SKOV3/CDDP/miR-199a-3p cells than that in mice injected with SKOV3/CDDP/miR-NC (Fig. 5D and E). In addition, the miR-199a-3p levels was significantly upregulated (Fig. 5F), while the ITGB8 expression was reduced in SKOV3/CDDP/miR-199a-3p xenografts (Fig. 5G and H). Taken together, these results indicate that miR-199a-3p enhances the CDDP sensitivity in ovarian cancer in vivo.

\section{Discussion}

miR-199a-3p was first identified in 2003 by computer analysis of mouse and Fugu rubripes sequences (13). Previous studies showed that miR-199a-3p was dysregulated in several types of cancers (14-17), including ovarian cancer (18-20), and may serve as a tumor suppressor in cancer development $(21,22)$. Recent studies have reported the essential role of miR-199a-3p in regulating drug resistance. For example, Li et al, reported that miR-199a-3p enhanced CDDP sensitivity of cholangiocarcinoma cells (23). Wang et al, demostreated that miR-199a could reverse cisplatin resistance in human ovarian cancer cells through the inhibition of mTOR (19). miR-199a-3p also regulated doxorubicin sensitivity of human hepatocarcinoma cells (24). However, the role and mechanism of miR-199a-3p involved in CDDP chemosensitivity in ovarian cancer remains largely unknown.

In the present study, we found significant downregulation of miR-199a-3p in chemoresistant ovarian cancer tissues, as well as CDDP-resistant cell line. Since miR-199a-3p downregulation was strongly related to CDDP resistant breast cancer and cholangiocarcinoma cells $(23,25)$, as well as ovarian cancer cells (19). miR-199a-3p may be involved in CDDP-resistance in ovarian cancer. Functional experiments demonstrated that forced miR-199-3p significantly reduced cell viability, G1 phase cell cycle arrest, cell invasion and promoted apoptosis in cisplatinresistant SKOV3/CDDP cells, compared to negative controls, whereas downregulation of miR-199a-3p showed the opposite effect, indicating that the role of $\mathrm{miR}-199 \mathrm{a}-3 \mathrm{p}$ in regulating cell growth, apoptosis, as well as metastasis in ovarian cancer may be correlated with the maintenance of CDDP sensitivity. However, the underlying molecular mechanism of miR-199a-3p in cisplatin resistance remains unclear.

We then analyzed the potential targets of miR-199a-3p and found that ITGB8 was significantly downregulated by miR-199a-3p. ITGB8 is a member of the integrin $\beta$-chain family and encodes a single-pass type I membrane protein with a VWFA domain and four cysteine-rich repeats (26). It binds 
to an $\alpha$ subunit to form a heterodimeric integrin complex (27). They also play critical roles in signal transduction that was involved in the regulation of cell growth and motility (28). In addition to cell motility, ITGB8 was recently reported associated with gefitinib resistance in hepatic cancer. Wang et al, showed that ITGB8 silencing decreased cell proliferation and increased gefitinib sensitivity in HepG2/G cells (29). Wala et al, showed that miR-199a-3p regulated ITGB8 expression in papillary renal cell carcinoma (17). In the present study, we showed a significantly negative correlation between ITGB8 and miR-199a-3p levels in ovarian cancer cells. In addition, no significant effect was observed in the luciferase activity of ITGB8 mutation between miR-199a-3p overexpressed and miR-199a-3p normally expressed cell lines, overexpression of miR-199a-3p remarkably decreased both mRNA and protein level of ITGB8 in SKOV3/CDDP cells, indicating that ITGB8 was a direct and functional target of miR-199a-3p.

In summary, the present study demonstrates, for the first time, that miR-199a-3p increases ovarian cancer cell sensitivity to CDDP by decreasing ITGB8 expression. The combination of miR-199a-3p with chemotherapy agents may prevent the development of drug resistance in ovarian cancer with CDDPresistance. However, the precise molecular mechanisms of miR-199a-3p, and the opportunities for its clinical use, require further investigation.

\section{Acknowledgements}

The present study was supported by the Programs for Science and Technology Development of Shaanxi Province (2016SF138).

\section{Competing interests}

The authors declare that they have no competing interests.

\section{References}

1. Siegel RL, Miller KD and Jemal A: Cancer statistics, 2015. CA Cancer J Clin 65: 5-29, 2015.

2. Jayson GC, Kohn EC, Kitchener HC and Ledermann JA: Ovarian cancer. Lancet 384: 1376-1388, 2014.

3. Cress RD, Chen YS, Morris CR, Petersen M and Leiserowitz GS: Characteristics of long-term survivors of epithelial ovarian cancer. Obstet Gynecol 126: 491-497, 2015.

4. Hammond SM: An overview of microRNAs. Adv Drug Deliv Rev 87: 3-14, 2015.

5. Eitan R, Kushnir M, Lithwick-Yanai G, David MB, Hoshen M, Glezerman M, Hod M, Sabah G, Rosenwald S and Levavi H: Tumor microRNA expression patterns associated with resistance to platinum based chemotherapy and survival in ovarian cancer patients. Gynecol Oncol 114: 253-259, 2009.

6. Yang N, Kaur S, Volinia S, Greshock J, Lassus H, Hasegawa K, Liang S, Leminen A, Deng S, Smith L, et al: MicroRNA microarray identifies Let-7i as a novel biomarker and therapeutic target in human epithelial ovarian cancer. Cancer Res 68: 10307-10314, 2008.

7. Tian S, Zhang M, Chen X, Liu Y and Lou G: MicroRNA-595 sensitizes ovarian cancer cells to cisplatin by targeting ABCB1. Oncotarget 7: 87091-87099, 2016.

8. Zhu X, Shen H, Yin X, Long L, Xie C, Liu Y, Hui L, Lin X, Fang Y, Cao Y, et al: miR-186 regulation of Twist1 and ovarian cancer sensitivity to cisplatin. Oncogene 35: 323-332, 2016.

9. van Jaarsveld MT, Helleman J, Boersma AW, van Kuijk PF, van Ijcken WF, Despierre E, Vergote I, Mathijssen RH, Berns EM, Verweij J, et al: miR-141 regulates KEAP1 and modulates cisplatin sensitivity in ovarian cancer cells. Oncogene 32: 4284-4293, 2013.
10. Li N, Yang L, Wang H, Yi T, Jia X, Chen C and Xu P: miR-130a and miR-374a function as novel regulators of cisplatin resistance in human ovarian cancer A2780 cells. PLoS One 10: e0128886, 2015.

11. Fu X, Tian J, Zhang L, Chen Y and Hao Q: Involvement of microRNA-93, a new regulator of PTEN/Akt signaling pathway, in regulation of chemotherapeutic drug cisplatin chemosensitivity in ovarian cancer cells. FEBS Lett 586: 1279-1286, 2012.

12. Zhang Y, Luo L, Zheng X and Yu T: An advanced orthotopic ovarian cancer model in mice for therapeutic trials. Biomed Res Int 2016: 2585787, 2016.

13. Lim LP, Lau NC, Weinstein EG, Abdelhakim A, Yekta S, Rhoades MW, Burge CB and Bartel DP: The microRNAs of Caenorhabditis elegans. Genes Dev 17: 991-1008, 2003.

14. Shatseva T, Lee DY, Deng Z and Yang BB: MicroRNA miR-199a-3p regulates cell proliferation and survival by targeting caveolin-2. J Cell Sci 124: 2826-2836, 2011.

15. Minna E, Romeo P, De Cecco L, Dugo M, Cassinelli G, Pilotti S, Degl'Innocenti D, Lanzi C, Casalini P, Pierotti MA, et al: miR-199a-3p displays tumor suppressor functions in papillary thyroid carcinoma. Oncotarget 5: 2513-2528, 2014

16. Liu C, Xing M, Wang L and Zhang K: miR-199a-3p downregulation in thyroid tissues is associated with invasion and metastasis of papillary thyroid carcinoma. Br J Biomed Sci 74: 90-94, 2017.

17. Wala SJ, Karamchandani JR, Saleeb R, Evans A, Ding Q, Ibrahim R, Jewett M, Pasic M, Finelli A, Pace K, et al: An integrated genomic analysis of papillary renal cell carcinoma type 1 uncovers the role of focal adhesion and extracellular matrix pathways. Mol Oncol 9: 1667-1677, 2015.

18. Chen R, Alvero AB, Silasi DA, Kelly MG, Fest S, Visintin I, Leiser A, Schwartz PE, Rutherford T and Mor G: Regulation of IKKbeta by miR-199a affects NF-kappaB activity in ovarian cancer cells. Oncogene 27: 4712-4723, 2008.

19. Wang Z, Ting Z, Li Y, Chen G, Lu Y and Hao X: microRNA-199a is able to reverse cisplatin resistance in human ovarian cancer cells through the inhibition of mammalian target of rapamycin. Oncol Lett 6: 789-794, 2013.

20. Wang Z, Liu Q, Gao Q, Luo Y, Li Q and Chen J: Involvement of miR-199a in cisplatin resistance of ovarian cancer cell through modulating expression of mTOR. Zhonghua Yi Xue Za Zhi 95: 2705-2708, 2015 (In Chinese).

21. Qu F, Zheng J, Gan W, Lian H, He H, Li W, Yuan T, Yang Y, Li X, Ji C, et al: MiR-199a-3p suppresses proliferation and invasion of prostate cancer cells by targeting Smad1. Oncotarget 8: 52465-52473, 2017.

22. Ghosh A, Dasgupta D, Ghosh A, Roychoudhury S, Kumar D, Gorain M, Butti R, Datta S, Agarwal S, Gupta S, et al: MiRNA199a-3p suppresses tumor growth, migration, invasion and angiogenesis in hepatocellular carcinoma by targeting VEGFA, VEGFR1, VEGFR2, HGF and MMP2. Cell Death Dis 8: e2706, 2017.

23. Li Q, Xia X, Ji J, Ma J, Tao L, Mo L and Chen W: MiR-199a-3p enhances cisplatin sensitivity of cholangiocarcinoma cells by inhibiting mTOR signaling pathway and expression of MDR1. Oncotarget 8: 33621-33630, 2017.

24. Fornari F, Milazzo M, Chieco P, Negrini M, Calin GA, Grazi GL, Pollutri D, Croce CM, Bolondi L and Gramantieri L: MiR-199a-3p regulates mTOR and c-Met to influence the doxorubicin sensitivity of human hepatocarcinoma cells. Cancer Res 70: 5184-5193, 2010.

25. Fan X, Zhou S, Zheng M, Deng X, Yi Y and Huang T: MiR-199a-3p enhances breast cancer cell sensitivity to cisplatin by downregulating TFAM (TFAM). Biomed Pharmacother 88 : 507-514, 2017.

26. LaPointe VL, Verpoorte A and Stevens MM: The changing integrin expression and a role for integrin $\beta 8$ in the chondrogenic differentiation of mesenchymal stem cells. PLoS One 8: e82035, 2013.

27. Cabodi S, Di Stefano P, Leal Mdel P, Tinnirello A, Bisaro B, Morello V, Damiano L, Aramu S, Repetto D, Tornillo G and Defilippi P: Integrins and signal transduction. Adv Exp Med Biol 674: 43-54, 2010.

28. Cabodi S, del Pilar Camacho-Leal M, Di Stefano P and Defilippi P: Integrin signalling adaptors: Not only figurants in the cancer story. Nat Rev Cancer 10: 858-870, 2010.

29. Wang WW, Wang YB, Wang DQ, Lin $Z$ and Sun RJ: Integrin beta-8 (ITGB8) silencing reverses gefitinib resistance of human hepatic cancer HepG2/G cell line. Int J Clin Exp Med 8: 3063-3071, 2015.

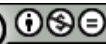

This work is licensed under a Creative Commons Attribution-NonCommercial-NoDerivatives 4.0 International (CC BY-NC-ND 4.0) License. 